\title{
A mathematical model for predicting glucose levels in critically-ill patients: the PIGnOLI model
}

Zhongheng Zhang

Background and objectives: Glycemic control is of paramount importance in the intensive care unit. Presently, several BG control algorithms have been developed for clinical trials, but they are mostly based on experts' opinion and consensus. There are no validated models predicting how glucose levels will change after initiating of insulin infusion in critically ill patients. The study aimed to develop an equation for initial insulin dose setting. Methods: A large critical care database was employed for the study. Linear regression model fitting was employed. Retested blood glucose was used as the independent variable. Insulin rate was forced into the model. Multivariable fractional polynomials and interaction terms were used to explore the complex relationships among covariates. Overall fit of the model was examined by using residuals and adjusted R-squared. Regression diagnostics were used to explore the influence of outliers on the model. Main results: A total of 6487 ICU admissions requiring insulin pump therapy were identified. The dataset was randomly split into two subsets at 7 to 3 ratio. The initial model comprised fractional polynomials and interactions terms. However, this model was not stable by excluding several outliers. We fitted a simple linear model without interaction. The selected prediction model (Predicting Glucose Levels in ICU, PIGnOLI) included variables of initial blood glucose, insulin rate, PO volume, total parental nutrition, body mass index (BMI), lactate, congestive heart failure, renal failure, liver disease, time interval of BS recheck, dextrose rate. Insulin rate was significantly associated with blood glucose reduction (coefficient: $-0.52,95 \% \mathrm{Cl}:-1.03,-0.01$ ). The parsimonious model was well validated with the validation subset, with an adjusted R-squared of 0.8259 . Conclusion: The study developed PIGnOLI model for the initial insulin dose setting. Furthermore, experimental study is mandatory to examine whether adjustment of insulin infusion rate based on PIGnOLI will benefit patients' outcomes. 


\section{A Mathematical Model for Predicting Glucose Levels in}

\section{Critically-Ill Patients: the PIGnOLI model}

4 Zhongheng ZHANG'1 (MMed);

5 Affiliation: Department of critical care medicine, Jinhua municipal central hospital, Jinhua

6 hospital of Zhejiang university, Zhejiang, P.R.China;

7 Corresponding author: Zhongheng ZHANG

8 Address: 351\#, Mingyue Road, Jinhua, Zhejiang province, China, 321000

9 Phone number: 86-579-82552667

10 Email: zh_zhang1984@hotmail.com

11 Key words: glycemic control, intensive care unit, insulin, big data, dosage.

13 There are no conflicts of interest. 


\section{Abstract}

Background and objectives: Glycemic control is of paramount importance in the intensive care unit. Presently, Several BG control algorithms have been developed for clinical trials, but they are mostly based on experts' opinion and consensus. There are no validated models predicting how glucose levels will change after initiating of insulin infusion in critically ill patients. The study aimed to develop an equation for initial insulin dose setting.

Methods: A large critical care database was employed for the study. Linear regression model fitting was employed. Retested blood glucose was used as the independent variable. Insulin rate was forced into the model. Multivariable fractional polynomials and interaction terms were used to explore the complex relationships among covariates. Overall fit of the model was examined by using residuals and adjusted R-squared. Regression diagnostics were used to explore the influence of outliers on the model.

Main results: A total of 6487 ICU admissions requiring insulin pump therapy were identified. The dataset was randomly split into two subsets at 7 to 3 ratio. The initial model comprised fractional polynomials and interactions terms. However, this model was not stable by excluding several outliers. We fitted a simple linear model without interaction. The selected prediction model (Predicting Glucose Levels in ICU, PIGnOLI) included variables of initial blood glucose, insulin rate, PO volume, total parental nutrition, body mass index (BMI), lactate, congestive heart failure, renal failure, liver disease, time interval of BS recheck, dextrose rate. Insulin rate was significantly associated with blood glucose reduction (coefficient: $-0.52,95 \% \mathrm{Cl}$ : -1.03 , 0.01 ). The parsimonious model was well validated with the validation subset, with an adjusted R-squared of 0.8259 .

Conclusion: The study developed PIGnOLI model for the initial insulin dose setting.

Furthermore, experimental study is mandatory to examine whether adjustment of insulin infusion rate based on PIGnOLI will benefit patients' outcomes. 


\section{Introduction}

42 Blood glucose (BG) control is of paramount importance in critically ill patients. A large body of evidence on BG control in intensive care unit (ICU) has emerged (1-3) and has lead to elaboration of international guidelines $(4,5)$, which state that both hypoglycemia and hyperglycemia are associated adverse outcomes. However, these guidelines simply give a target of BG to achieve without elaborating on specific algorithms to achieve such a target range.

There are many algorithms on the dosing of insulin to control BG. In the well-known NICESUGAR study (6), specific protocol on the dosing of insulin was given, aiming to reach a steady BS within target ranges in both aims. This protocol categorized dosing strategies on whether insulin was first initiated or continued. In another study conducted in Australia, a locally developed protocol was found to be effective in maintaining BG in target range (7). However, several common features of these protocols include: 1) they were developed largely by expert opinion and experiences. These experts can be nurses, pharmacists, intensivists and investigators; 2 ) they only take into account a limited number of clinical variables such as the measured BG and the trend of BG changing after initiation of insulin pump. However, there are numerous factors that can influence insulin sensitivity. These factors include but are not limited to the history of diabetes, severity of illness, liver function and route of glucose intake.

It is important in critically ill patients to have the ability to predict response to medications. Regression modeling has been used to model predicted drug response. Medication dosing by using this approach is useful for drugs that have narrow therapeutic window and require frequent dosing adjustment to reach a predefined target range. In critical care setting, heparin dosing is a good example and has been investigated by using this regression modeling approach 
66

67

68

69

mathematical model predicting the change in glucose level resulting from the initiation of insulin infusion in critically ill patients.

\section{Methods}

\section{Design}

The retrospective study encompassed analysis of a Multiparameter Intelligent Monitoring in Intensive Care II (MIMIC-II), a large clinical database of critically ill patients. Because the study utilized an open access clinical database, formal IRB approval was not required.

\section{Data source}

MIMC-II is a large registry of intensive care unit patients treated at Beth Israel Deaconess Medical Center, Boston, Massachusetts. Patients' information on demographics, laboratory findings, imaging study, vital signs and progress notes were available (8). MIMIC contains data on over 30,000 patients admitted during the period 2001-2008. The database comprised varieties of ICUs, including the medical, surgical, coronary, and cardiac surgery recovery care units. ICU stays separated by less than 24 hours were considered as one episode of ICU stay. Data were collected from electronic health data and all information produced during hospital stay were stored in the database.

All data were extracted by using structural query language (SQL) programming language from the database (9). The Institutional Review Boards of the Massachusetts Institute of Technology (Cambridge, MA) and Beth Israel Deaconess Medical Center (Boston, MA) approved the establishment of the database. De-identification was performed to ensure patients' confidentiality. Access to the database was approved after completion of the NIH web-based 
92

113 Simultaneous use of intravenous (IV) total parental nutrition (TPN) and dextrose were extracted

114 from the database. Different concentrations of dextrose were transformed to $5 \%$ dextrose (e.g. 115

training course named "Protecting Human Research Participants" by the author Z.Z. (certification number: 1132877).

\section{Selection of Subjects}

All adult patients were considered potentially eligible for our study. Patients actually received continuous insulin infusion were included. Children and neonates were excluded.

\section{Outcomes}

The primary outcome was retested BG level (mg/dl). The value, date and time of each BG were recorded in medical record. BG values can be those from blood chemistry and fingerstick, and the differentiation between venous glucose measurements and fingerstick levels was not performed in current analysis.

\section{Clinical Variables}

Specific SQL programming languages for data extraction are shown in supplemental file (Supplemental Digital Content_1_data extraction). Comorbidities including diabetes, liver failure, congestive heart failure and renal failure were extracted because we felt that they may influence the sensitivity to insulin therapy. Laboratory parameters including bilirubin, C-reactive protein, serum creatinine and lactate were extracted. A total number of 1,117,076 BG measured with finger stick and in chemistry were extracted. A total number of 480,560 episodes of insulin rate were extracted.

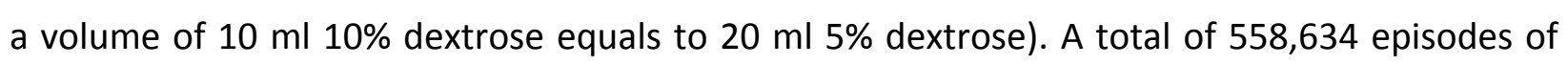
oral feeding (PO) containing glucose were extracted for its volume and time. All events were based upon charted time. 


\section{Data analysis}

The objective of the analysis was to establish a linear regression equation between retested BG and insulin rate, controlling for other potential confounders. A data-driven approach means that the form of the equation was determined by data, depending on statistical significance. All variables thought to be associated with insulin sensitivity were extracted from the database and were considered for their inclusion in the model at outset.

I employed multivariable fractional polynomial (MFP) method to construct the main effect model. The method combines backward elimination of statistically non-significant covariates with an iterative examination of the scale of continuous variables. MFP specifies two levels of significance levels: $\alpha 1=0.15$ for the test for exclusion and addition of variables to the equation and $\alpha 2=0.05$ to assess significance of fractional polynomial transforms of continuous variables. One degree of freedom was assigned to dichotomous variables and two-term fractional polynomials with 4 degrees of freedom were assigned to continuous variables. Continuous variables were modeled using closed test procedure, determining whether the covariate should be dropped from model at $\alpha 1$. Then $\alpha 2=0.05$ was employed to test the need for transformation of the variable. With closed test procedure, the best two-term transformation was compared to the linear term. If two term model is significantly better than the linear one at $\alpha 2=0.05$, two term model is then compared to one-term model. Otherwise, linear term was retained in the model. Interactions were explored and terms with $p<0.05$ were retained in the model.

The overall fit of the model was assessed by using R-squared which is a reflection of the variance that can be explained by the model. Influential observations were evaluated by examining the leverage, Cook's D and DFITS. Influential observations were excluded and the model was refitted by using MFPIGEN module. If the new model was significantly different from the original one, the original model would be reconsidered for more parsimonious one. For example, some fractional transformation would be dropped and interaction terms could be 
dropped if the likelihood ratio test showed $p>0.05$. R-squared of the new parsimonious model would be compared to the original one to see whether the fitness was good enough. The whole dataset was split into two subsets, the training subset and the validation subset. Observed values of covariates were substituted into the fitted model to derive linear prediction. We then performed regression model with linear prediction of the training subset as dependent variable and linear prediction of the validation subset as independent variable. The regression coefficient should be close to 1 and statistically significant at $p<0.05$ if the model fits well to the validation subset.

All statistical analyses were performed by using Stata 13.1 (StataCorp College Station, TX 77845, USA) and R software (R 3.1.1). Statistical significance was considered at $p<0.05$.

\section{Results}

A total of 6487 ICU admissions requiring insulin pump therapy were identified from the dataset. The dataset was randomly split into two subsets at 7 to 3 ratio. The training subset comprised 4593 observations and the validation subset comprised 1894 observations.

\section{Model exploration and development}

The results of initial model fitting are shown in table 1 . The continuous variables including glucose, interval, dextrose rate and insulin rate were FP transformed and there were significant interactions between insulin rate and two terms of glucose. Glucose was transformed by twoterm FP with the power of -0.5 and 1 . Interval was transformed by two-term FP with the power of -2 and 1. Dextrose rate was transformed by one term FP with the power of 0.5 . There were two interaction terms between insulin rate and glucose because glucose was modeled with two terms. The overall fit of the model was thought to be good with an adjusted R-squared of 0.8449 . 
171 Influential observations were examined by using regression diagnostics (Supplemental Digital 172 Content_2_diagnostics). By excluding these influential observations, we refitted the model and 173 found that FP terms and coefficients were remarkably changed (table 2). Glucose was 174 transformed by two-term FP with the power of -2 and 1. Interval was transformed by two-term

power of 3 and 3 . The results showed that the model was not stable, probably due to complexity of the FP assignment and multiple testing during model fitting. The FP terms were influenced by several influential observations.

Parsimonious model was fitted to address the problem of instability. Graphical presentation showed that although the interaction term was statistically significant, the magnitude was of marginal clinical significance (figure 3 in Supplemental Digital Content_2_diagnostics). Therefore, we opted not to incorporate interaction terms in the parsimonious model. Figure 1 shows the scatter points predicted by FP model and simple linear model, and the two lines were close to each other. Visual inspection of the graph indicates the use of parsimonious model would not compromise the prediction accuracy of the model.

\section{Final model and model validation}

The final Predicting Glucose Levels in ICU (PIGnOLI) model is shown in table 3. Insulin rate was significantly associated with blood glucose reduction (coefficient: $-0.52,95 \% \mathrm{Cl}:-1.03,-0.01$ ). Initial blood glucose was the most important determinant of retested blood glucose (coefficient: 0.89, 95\% $\mathrm{Cl}: 0.88,0.90)$. Oral intake, TPN and dextrose infusion were all associated with blood glucose control. Furthermore, serum lactate and BMI were positively associated with retested blood glucose. Time interval was negatively associated with retested blood glucose level (coefficient: $-0.18 ; 95 \% \mathrm{Cl}:-0.22,-0.14)$. The PIGnOLI model showed an adjusted R-squared of 0.84, which was not significantly different from the FP model with interaction terms (Rsquared=0.84). PIGnOLI model was tested in the validation subset and the result showed that the coefficient between estimated retest glucose and observed retest glucose was 0.99 (95\% $\mathrm{Cl}: 0.97-1.01 ; \mathrm{p}<0.001)$. The adjusted R-squared was 0.8259 , suggesting that the model was well 
calibrated with the validation subset.

\section{Discussion}

This study developed the PIGnOLI model for BG control in critically ill patients. A data-driven approach could be applied in our study because there is large volume of retrospective data available for analysis. The widespread uses of electronic medical record systems have made this strategy possible. The present study provides a framework for predicting and modeling BG response. This approach may be useful for predicting medication response in this and other disease states.

Although there is large body of evidence suggesting the importance of BG control in the intensive care unit (ICU), there is no empirical data on how to control BG (10). Several BG control algorithms have been developed for clinical trials, but they are mostly based on experts' opinion and consensus. As a result, many patients assigned to a specific BG range cannot reach that range, or many times insulin rate adjustment are required before an optimal target is reached. Furthermore, substantial number of patients experience under- or over-control of BG because of insulin misuse and/or other disease-related factors. It is optimal in clinical practice that we can accurately control BG within a short period of time. In the present study, we developed an equation for insulin adjustment, by considering comorbidities, laboratory findings and demographics. Glucose intakes such as TPN, dextrose infusion and PO intake during the analysis time were all considered. In critical care practice, these information need to be collected and put into calculators to estimate the initial rate of insulin infusion. Since these variables are routinely recorded in most ICUs, this could be done with just any electronic health record (HER) system.

Glycemic control in the present clinical practice is not based on data-driven approach. For 
example, in the well-known NICE-SUGAR study (6), insulin dosing algorithm was based on whether insulin was first initiated or continued. Insulin rate was determined on the value of BG, taking previous BG into consideration. This protocol did not take into account of other variables such as concomitant dextrose infusion, baseline renal and liver functions. In another study conducted in Australia, a locally developed protocol was found to be effective in maintaining BG in target range (7). The insulin rate was set according to the amount of BG fall, without considering other potential influential factors.

The predictors in the PIGnOLI model have biologic and clinical plausibility. For example, congestive heart failure was positively associated with blood glucose. In a cohort of 3,748 nondiabetic participants aged $\geq 65$ years, Guglin $M$ and coworkers(11) found that baseline heart failure was associated with subsequent development of diabetes mellitus within 3-4 years. Liver disease may contribute to hyperglycemia via insulin resistance and increase hepatic BG output $(12,13)$. With respect to the association of renal failure with glycemic control, although the present study failed to found a significant association at $p=0.05$, we still incorporated this factor into our model because renal function has been identified to be tightly related to BG levels (14). Serum lactate is a biomarker of tissue perfusion, and it increases markedly with hypoperfusion and hypoxia. Our previous work has demonstrated that lactate is a strong predictor of clinical outcome in critically ill patients (15-17). We propose that since lactate is biomarker of circulatory shock, it is also a biomarker of stress response during severe illness. Stress response is a well-established contributor to insulin resistance and observed hyperglycemia (18).

Many drugs require careful dosing because their therapeutic and toxic doses are close to each other. Insulin is one of such drug that its therapeutic dose varies substantially across individual patients. More importantly, inappropriate dosing may cause catastrophic consequences such as infection, permanent neurologic defect and coma. Therefore, close monitoring of BG and frequent adjustment of insulin dose are mandatory. Due to the complexity of PIGnOLI model 
251

252

that may hinder its use in clinical practice, I programed PIGnOLI model in Excel format (Supplemental Digital Content_3_PIGnOLI) to ease its use (figure 2). The users can input required variables and predict retested BG after predefined time interval (<120 min).

Several limitations need to be acknowledged in our study. The study restricted to dosing at the initiation of insulin pump and subsequent adjustment was not addressed. The difficulty lay in the complexity of data preprocessing. In future study I will try to resolve these technical difficulties and provide further algorithms on how to adjust insulin dose by incorporating initial response to insulin therapy in addition to covariates as reported in the present study. This analysis included only patients receiving insulin via pump and would not necessarily be generalizable to patients receiving insulin by some other route. However, insulin pump is the most attractive mean to give insulin for critically ill patients, mostly due to its accuracy in dosing and the property of short acting. Our study may suffer from the problem of multiple testing and model overfitting. This happened in our first model, in which several FP terms and complex interactions were incorporated. However, this model was found to be unstable by excluding several outliers. Therefore, we opted to employ simple linear terms and clinical irrelevant interactions were excluded. The PIGnOLI model was validated in split subset and was well fitted to the independent subset. One important limitation of the study is that the database is limited to one center, and thus the extrapolation of the PIGnOLI model to other systems and countries requires further validation.

In conclusion, the study developed PIGnOLI model for the initial insulin dose setting. It may be favorable if this algorithm can be used in clinical setting for accurate BG control for critically ill patients. Furthermore, experimental study is mandatory to examine whether insulin adjustment based on PIGnOLI model will benefit patients' outcomes. Before we can use PIGnOLI model in clinical practice, it is also mandatory to compare the episodes of hypoglycemia and duration of hyperglycemia between groups using and without using 


\section{PIGnOLI model.}

279

280

Peer] reviewing PDF | (2015:04:4647:2:0:NEW 16 May 2015) 


\section{Figure legends}

283 Figure 1. Graphical presentation of the BG predicted by the model including FP terms (red line) and

284 the model with linear terms (blue line). Both models appeared similar in predicting BG. The 285 initial BG was controlled at its mean value of $195.9 \mathrm{mg} / \mathrm{dl}$.

286 Figure 2. A snapshot of the calculator for setting initial dose of insulin.

287

288 


\section{Reference}

290 1. Arabi YM, Dabbagh OC, Tamim HM, Al-Shimemeri AA, Memish ZA, Haddad SH, et al. Intensive versus

291 conventional insulin therapy: a randomized controlled trial in medical and surgical critically ill patients. Critical care 292 medicine. 2008;36(12):3190-7.

293 2. Griesdale DE, de Souza RJ, van Dam RM, Heyland DK, Cook DJ, Malhotra A, et al. Intensive insulin therapy and 294 mortality among critically ill patients: a meta-analysis including NICE-SUGAR study data. CMAJ : Canadian Medical 295 Association journal = journal de l'Association medicale canadienne. 2009;180(8):821-7.

296 3. van den Berghe G, Wouters P, Weekers F, Verwaest C, Bruyninckx F, Schetz M, et al. Intensive insulin therapy 297 in critically ill patients. The New England journal of medicine. 2001;345(19):1359-67.

298 4. Ichai C, Cariou A, Leone M, Veber B, Barnoud D, le Groupe dE. [Expert's formalized recommendations. 299 Glycemic control in ICU and during anaesthesia: useful recommendations]. Annales francaises d'anesthesie et de 300 reanimation. 2009;28(7-8):717-8.

301 5. Qaseem A, Humphrey LL, Chou R, Snow V, Shekelle P, Clinical Guidelines Committee of the American College 302 of $P$. Use of intensive insulin therapy for the management of glycemic control in hospitalized patients: a clinical 303 practice guideline from the American College of Physicians. Annals of internal medicine. 2011;154(4):260-7.

304 6. Investigators N-SS, Finfer S, Chittock DR, Su SY, Blair D, Foster D, et al. Intensive versus conventional glucose 305 control in critically ill patients. The New England journal of medicine. 2009;360(13):1283-97.

306 7. Breeding J, Welch S, Buscher H, Nair P, Frost C, Newman S, et al. A retrospective audit of insulin infusion 307 management involving a locally developed dynamic insulin infusion guideline in a tertiary ICU. Australian critical 308 care : official journal of the Confederation of Australian Critical Care Nurses. 2014.

309 8. Saeed M, Villarroel M, Reisner AT, Clifford G, Lehman LW, Moody G, et al. Multiparameter Intelligent 310 Monitoring in Intensive Care II: a public-access intensive care unit database. Critical care medicine. 2011;39(5):95231160.

312 9. Zhang Z. Accessing critical care big data: a step by step approach. Journal of thoracic disease. 2015;7(3):23831342.

314 10. Fahy BG, Sheehy AM, Coursin DB. Glucose control in the intensive care unit. Critical care medicine. 315 2009;37(5):1769-76.

316 11. Guglin M, Lynch K, Krischer J. Heart failure as a risk factor for diabetes mellitus. Cardiology. 2014;129(2):84-92.

317 12. DeFronzo RA. Lilly lecture 1987. The triumvirate: beta-cell, muscle, liver. A collusion responsible for NIDDM. 318 Diabetes. 1988;37(6):667-87.

319 13. Mitrakou A, Kelley D, Veneman T, Jenssen T, Pangburn T, Reilly J, et al. Contribution of abnormal muscle and 320 liver glucose metabolism to postprandial hyperglycemia in NIDDM. Diabetes. 1990;39(11):1381-90.

321 14. DeFronzo RA, Davidson JA, Del Prato S. The role of the kidneys in glucose homeostasis: a new path towards 322 normalizing glycaemia. Diabetes, obesity \& metabolism. 2012;14(1):5-14.

323 15. Zhang Z, Chen K, Ni H, Fan H. Predictive value of lactate in unselected critically ill patients: an analysis using 324 fractional polynomials. Journal of thoracic disease. 2014;6(7):995-1003.

325 16. Zhang Z, Xu X. Lactate clearance is a useful biomarker for the prediction of all-cause mortality in critically ill 326 patients: a systematic review and meta-analysis*. Critical care medicine. 2014;42(9):2118-25.

327 17. Zhang Z, Xu X, Chen K. Lactate clearance as a useful biomarker for the prediction of all-cause mortality in 328 critically ill patients: a systematic review study protocol. BMJ open. 2014;4(5):e004752. 
329 18. Santos L. Stress response in critical illness. Current problems in pediatric and adolescent health care. 330 2013;43(10):264-72.

331

332 


\section{Table 1 (on next page)}

Table 1 Multivariable linear regression model to predict retested blood glucose $(\mathrm{mg} / \mathrm{dl})$ after initiation of insulin infusion

Table1 
2 Table 1 Multivariable linear regression model to predict retested blood glucose $(\mathrm{mg} / \mathrm{dl})$ 3 after initiation of insulin infusion

\begin{tabular}{|c|c|c|c|c|c|}
\hline Covariates $^{\S}$ & Coefficient & $\begin{array}{l}\text { Standard } \\
\text { error }\end{array}$ & $\begin{array}{l}\text { Lower limit } \\
\text { of } 95 \% \mathrm{CI}\end{array}$ & $\begin{array}{l}\text { Upper limit } \\
\text { of } 95 \% \mathrm{CI}\end{array}$ & $\mathrm{p}$ \\
\hline Glucose $\mathrm{e}^{-0.5}$ & 34.33 & 8.81 & 17.07 & 51.59 & $<0.001$ \\
\hline $\begin{array}{l}\text { Glucose- } \\
1.96\end{array}$ & 94.00 & 1.60 & 90.85 & 97.14 & $<0.001$ \\
\hline $\begin{array}{l}\text { (Insulin } \\
\text { rate-2.85) }\end{array}$ & -1.06 & 0.33 & -1.70 & -0.42 & $<0.001$ \\
\hline $\begin{array}{l}\text { (time } \\
\text { interval) }{ }^{2}- \\
8.13\end{array}$ & -0.002 & 0.0006 & -0.004 & -0.001 & $<0.001$ \\
\hline $\begin{array}{l}\text { time } \\
\text { interval - } \\
0.35\end{array}$ & -18.90 & 2.30 & -23.41 & -14.39 & $<0.001$ \\
\hline $\begin{array}{l}\text { (dextrose } \\
\text { rate) }\end{array}$ & 22.02 & 8.58 & 5.20 & 38.84 & 0.01 \\
\hline PO volume & -0.02 & 0.01 & -0.03 & 0.00 & 0.06 \\
\hline $\begin{array}{l}\text { TPN } \\
\text { volume }\end{array}$ & 0.07 & 0.03 & 0.02 & 0.12 & 0.01 \\
\hline $\begin{array}{l}\text { Lactate } \\
(\mathrm{mmol} / \mathrm{l})\end{array}$ & 0.87 & 0.23 & 0.41 & 1.32 & 0.00 \\
\hline $\begin{array}{l}\text { History of } \\
\text { congestive } \\
\text { heart } \\
\text { failure }\end{array}$ & 2.64 & 1.18 & 0.33 & 4.95 & 0.03 \\
\hline $\begin{array}{l}\text { History of } \\
\text { renal } \\
\text { failure }\end{array}$ & -3.13 & 1.87 & -6.78 & 0.53 & 0.09 \\
\hline $\begin{array}{l}\text { History of } \\
\text { liver } \\
\text { disease }\end{array}$ & 4.50 & 2.03 & 0.52 & 8.47 & 0.03 \\
\hline $\begin{array}{l}\text { (glucose- } \\
0.5) \times \\
\text { (Insulin } \\
\text { rate-2.85) }\end{array}$ & 10.92 & 4.34 & 2.41 & 19.43 & 0.01 \\
\hline $\begin{array}{l}\text { (Glucose- } \\
1.96)^{\times} \\
\text {(Insulin } \\
\text { rate-2.85) }\end{array}$ & 1.60 & 0.67 & 0.28 & 2.91 & 0.02 \\
\hline Constant & 186.52 & 0.98 & 184.61 & 188.44 & $<0.001$ \\
\hline
\end{tabular}

4 Number of obs $=4593, F(14,4578)=1787.14$, Prob $>F=0.0000$, R-squared $=0.8453$, Adj R-

5 squared $=0.8449$, Root MSE $=30.569$. 
6 \$Some covariates were centered and transformed with fractional polynomials.

7 Abbreviations: PO: by mouth, orally (from the Latin "per os", by mouth); TPN: total 8 parental nutrition. 


\section{Table 2 (on next page)}

Table 2 refitting the regression model after excluding influential observations

Table2 
2 Table 2 refitting the regression model after excluding influential observations

\begin{tabular}{|c|c|c|c|c|c|}
\hline Covariates $^{\S}$ & $\begin{array}{l}\text { Coefficie } \\
\text { nt }\end{array}$ & $\begin{array}{l}\text { Standar } \\
\text { d error }\end{array}$ & $\begin{array}{l}\text { Lower } \\
\text { limit of } \\
95 \% \mathrm{CI}\end{array}$ & $\begin{array}{l}\text { Upper } \\
\text { limit of } \\
95 \% \text { CI }\end{array}$ & $\mathrm{p}$ \\
\hline (glucose $/ 100)^{-2}$ & 10.865 & 2.589 & 5.789 & 15.941 & $\begin{array}{l}<0.00 \\
1\end{array}$ \\
\hline (glucose/100)-1.96 & 92.193 & 0.985 & 90.261 & 94.125 & $\begin{array}{l}<0.00 \\
1\end{array}$ \\
\hline insulin rate- 2.85 & -0.861 & 0.316 & -1.481 & -0.241 & 0.007 \\
\hline$(\text { interval/100) })^{3}$ & -5.530 & 7.977 & $\begin{array}{l}- \\
21.168 \\
\end{array}$ & 10.109 & 0.488 \\
\hline $\begin{array}{l}\text { (interval/100) } 3 \times \ln (\text { interval } / \\
100)\end{array}$ & 77.272 & 19.426 & 39.189 & $\begin{array}{l}115.35 \\
6\end{array}$ & $\begin{array}{l}<0.00 \\
1\end{array}$ \\
\hline $\begin{array}{l}{\left[(\text { dextrose rate+0.01)/100] }]^{0.5}\right.} \\
9.3 \times 10^{-6}\end{array}$ & 5.584 & 1.703 & 2.247 & 8.922 & 0.001 \\
\hline $\begin{array}{l}{[(\text { dextrose rate+0.01)/100] }} \\
\times \ln [(\text { dextrose } \\
\text { rate }+0.01) / 100]+3.6 \times 10^{-5}\end{array}$ & -4.011 & 1.106 & -6.180 & -1.843 & $\begin{array}{l}<0.00 \\
1\end{array}$ \\
\hline PO volume & -0.013 & 0.008 & -0.029 & 0.002 & 0.094 \\
\hline TPN volume & 0.091 & 0.030 & 0.031 & 0.150 & 0.003 \\
\hline Lactate $(\mathrm{mmol} / \mathrm{l})$ & 0.865 & 0.227 & 0.421 & 1.310 & $\begin{array}{l}<0.00 \\
1\end{array}$ \\
\hline Congestive heart failure & 2.376 & 1.151 & 0.120 & 4.632 & 0.039 \\
\hline Renal failure & -2.983 & 1.817 & -6.546 & 0.580 & 0.101 \\
\hline Liver disease & 4.254 & 1.977 & 0.379 & 8.129 & 0.031 \\
\hline $\begin{array}{l}\text { (glucose } / 100)^{-2} \times(\text { insulin } \\
\text { rate-2.85) }\end{array}$ & 0.489 & 1.163 & -1.792 & 2.769 & 0.674 \\
\hline $\begin{array}{l}{[(\text { glucose } / 100)-1.96] \times(} \\
\text { insulin rate-2.85) }\end{array}$ & 0.189 & 0.358 & -0.513 & 0.891 & 0.598 \\
\hline Constant & 185.875 & 0.910 & $\begin{array}{l}184.09 \\
1\end{array}$ & $\begin{array}{l}187.65 \\
9\end{array}$ & $\begin{array}{l}<0.00 \\
1\end{array}$ \\
\hline
\end{tabular}

3 Number of obs $=4585, F(15,4569)=1760.88$, Prob $>F=0.0000$, R-squared $=0.88525$, Adj

4 R-squared $=0.8520$, Root MSE $=29.784$

5 \$Some covariates were centered and transformed with fractional polynomials.

6 Abbreviations: PO: by mouth, orally (from the Latin "per os", by mouth); TPN: total

7 parental nutrition. 


\section{Table 3(on next page)}

Table 3 Parsimonious model with linear terms and no interaction 
3 Table 3 Parsimonious model with linear terms and no interaction

\begin{tabular}{|l|l|l|l|l|l|}
\hline Covariates & Coefficient & $\begin{array}{l}\text { Standard } \\
\text { error }\end{array}$ & $\begin{array}{l}\text { Lower limit } \\
\text { of 95\% CI }\end{array}$ & $\begin{array}{l}\text { Upper limit } \\
\text { of 95\% CI }\end{array}$ & $\mathrm{p}$ \\
\hline Insulin rate & -0.52 & 0.26 & -1.03 & -0.01 & 0.05 \\
\hline Glucose & 0.89 & 0.01 & 0.88 & 0.90 & $<0.001$ \\
\hline PO volume & -0.02 & 0.01 & -0.03 & -0.00 & 0.05 \\
\hline $\begin{array}{l}\text { TPN } \\
\text { volume }\end{array}$ & 0.07 & 0.03 & 0.01 & 0.12 & 0.01 \\
\hline BMI & 0.10 & 0.06 & -0.02 & 0.22 & 0.09 \\
\hline $\begin{array}{l}\text { Lactate } \\
\text { (mmol/l) }\end{array}$ & 0.95 & 0.23 & 0.50 & 1.41 & $<0.001$ \\
\hline $\begin{array}{l}\text { Congestive } \\
\text { heart } \\
\text { failure }\end{array}$ & 2.58 & 1.19 & 0.26 & 4.91 & 0.03 \\
\hline $\begin{array}{l}\text { Renal } \\
\text { failure }\end{array}$ & -3.09 & 1.87 & -6.76 & 0.58 & 0.10 \\
\hline $\begin{array}{l}\text { Liver } \\
\text { disease }\end{array}$ & 4.13 & 2.03 & 0.14 & 8.12 & 0.04 \\
\hline Interval & -0.18 & 0.02 & -0.22 & -0.14 & $<0.001$ \\
\hline $\begin{array}{l}\text { Dextrose } \\
\text { rate (5\%) }\end{array}$ & 0.01 & 0.01 & -0.01 & 0.03 & 0.20 \\
\hline Constant & 17.18 & 2.23 & 12.82 & 21.55 & $<0.001$ \\
\hline
\end{tabular}

4 Number of obs $=4593, \mathrm{~F}(11,4581)=2251.71$, Prob $>\mathrm{F}=0.0000$, R-squared $=0.8439$, Adj R-

5 squared $=0.8435$, Root $\mathrm{MSE}=30.698$

6 Abbreviations: PO: by mouth, orally (from the Latin "per os", by mouth); TPN: total

7 parental nutrition; BMI: body mass index. 
Figure 1 (on next page)

figure 1

Figure 1. Graphical presentation of the BG predicted by the model including FP terms (red line) and the model with linear terms (blue line). Both models appeared similar in predicting BG. The initial BG was controlled at its mean value of $195.9 \mathrm{mg} / \mathrm{dl}$. 


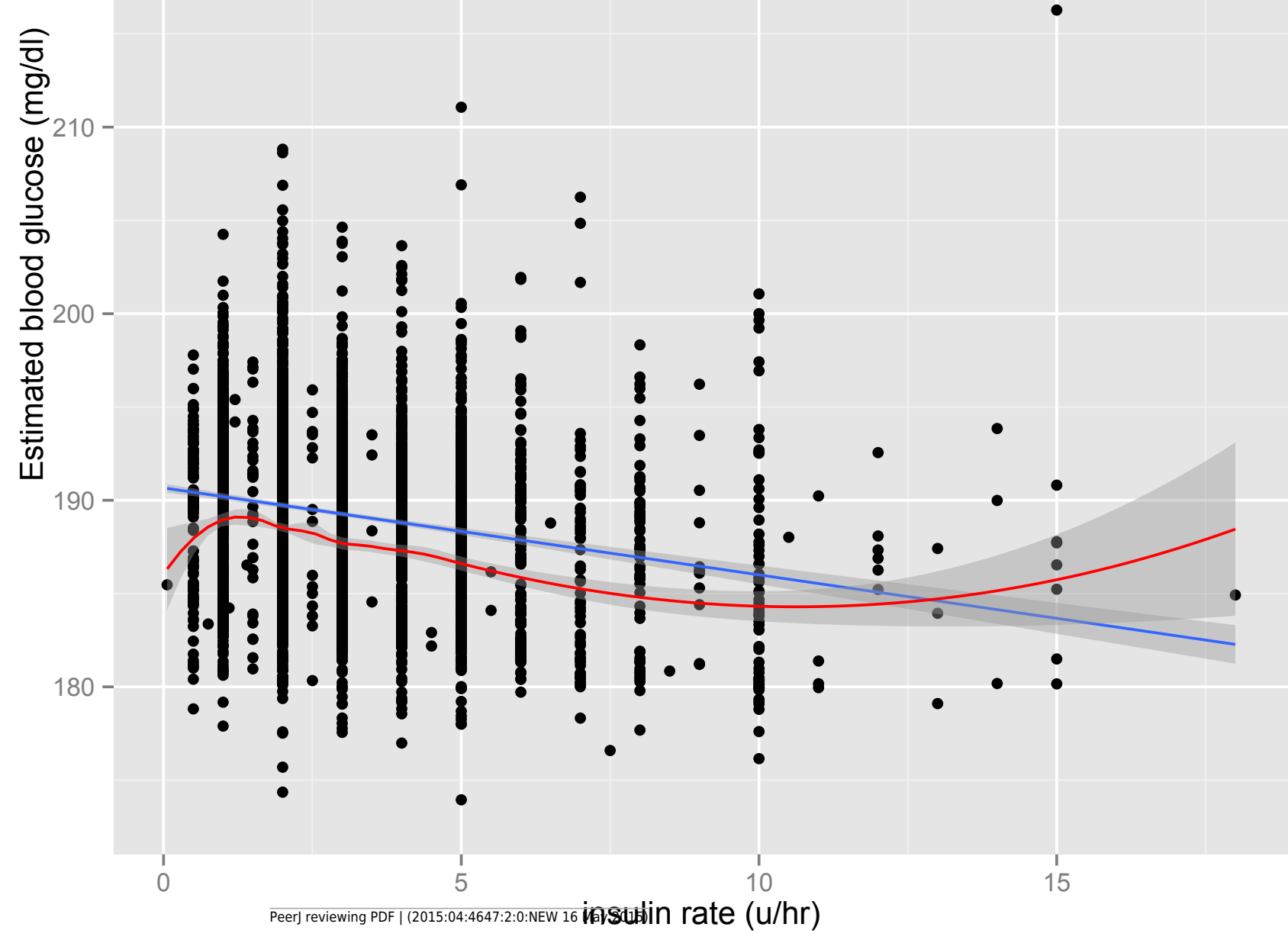




\section{2}

\section{Figure 2}

Figure 2. A snapshot of the calculator for setting initial dose of insulin.

\section{Blood glucose control in the intensive care unit}

\begin{tabular}{|c|c|c|}
\hline Variables & Values & Note: \\
\hline Glucose (mg/dl) & 400 & The initial blood glucose level $(<500 \mathrm{mg} / \mathrm{dl})$ \\
\hline PO volume (ml) & 100 & The amount of oral intake volume \\
\hline $\mathrm{BMI}(\mathrm{kg} / \mathrm{m} 2)$ & 23 & Body mass index \\
\hline Lactate (mmol/l) & 4.3 & \\
\hline Liver disease & 1 & "1" to indicate the patient has this comorbidity; otherwise value of " 0 " is assigned \\
\hline Time interval of BS recheck & 120 & The value should be within 120 minutes \\
\hline Dextrose rate $(\mathrm{ml} / \mathrm{hr})$ & 30 & Convert to equivalent volume of $5 \%$ Dextrose \\
\hline Retest BS (mg/dl) & 356.485 & Recheck blood glucose \\
\hline
\end{tabular}

